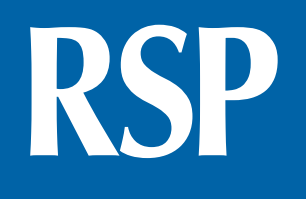

http://www.rsp.fsp.usp.br/

Revista de Saúde Pública

\title{
Tendências na mortalidade por câncer de próstata no estado de São Paulo, 2000 a 2015
}

\author{
Carolina Terra de Moraes Luizaga' (iD, Karina Braga Ribeiro" iD, Luiz Augusto Marcondes \\ Fonseca"II iD, José Eluf Neto ${ }^{\mathrm{IV}, \mathrm{V}}$ iD \\ I Fundação Oncocentro de São Paulo. Diretoria de Informação e Epidemiologia. São Paulo, SP, Brasil \\ " Faculdade de Ciências Médicas da Santa Casa de São Paulo. Departamento de Saúde Coletiva. São Paulo, \\ SP, Brasil \\ III Universidade de São Paulo. Faculdade de Medicina. Hospital das Clínicas. Serviço de Imunologia Clínica e \\ Alergia. São Paulo, SP, Brasil \\ Iv Universidade de São Paulo. Faculdade de Medicina. Departamento de Medicina Preventiva. São Paulo, \\ SP, Brasil \\ v Fundação Oncocentro de São Paulo. São Paulo, SP, Brasil
}

\author{
Correspondência: \\ Carolina Terra de Moraes Luizaga \\ Fundação Oncocentro de São Paulo \\ Diretoria de Informação e \\ Epidemiologia \\ Rua Oscar Freire, $23966^{\circ}$ andar \\ 05409-012 São Paulo, SP, Brasil \\ E-mail: carolinaterra.ml@gmail.com \\ Recebido: 22 jul 2019 \\ Aprovado: 4 dez 2019 \\ Como citar: Luizaga CTM, \\ Ribeiro KB, Fonseca LAM, Neto \\ JE. Tendências na mortalidade por \\ câncer de próstata no estado de \\ São Paulo, 2000 a 2015. Rev Saude \\ Publica. 2020;54:87.
}

Copyright: Este é um artigo de acesso aberto distribuído sob os termos da Licença de Atribuição Creative Commons, que permite uso irrestrito, distribuição e reprodução em qualquer meio, desde que o autor e a fonte originais sejam creditados.

\section{RESUMO}

OBJETIVO: Estimar a magnitude e identificar padrões de mudança na mortalidade por câncer de próstata no estado de São Paulo e nas 17 redes regionais de atenção à saúde, segundo grupos etários a partir dos 50 anos, no período de 2000 a 2015.

MÉTODOS: As taxas de mortalidade ajustadas por idade (por 100 mil homens) foram calculadas pelo método direto usando a população mundial de Segi como padrão. A análise de regressão Joinpoint foi utilizada para calcular as variações percentuais anuais médias (AAPC), com intervalo de confiança de 95\% (IC95\%), por rede regional e grupo etário (50-59, 60-69, 70-79 e 80 anos ou mais).

RESULTADOS: Para o estado de São Paulo, as taxas ajustadas de mortalidade foram de 15,2, 13,3 e 11,9/100 mil homens, respectivamente, nos períodos de 2000 a 2005, 2006 a 2010 e 2011 a 2015, com tendência de decréscimo significativo (AAPC $=-2,10 \%$; IC95\% $-2,42--1,79$ ) a cada ano. Das 17 redes, 11 apresentaram reduções médias anuais significativas, que variaram entre $-1,72 \%$ e $-3,05 \%$. A partir dos 50 anos, verificou-se redução mais acentuada nos grupos de 50 a 59 (AAPC = -2,33\%; IC95\% -3,04 - -1,62) e 60 a 69 anos (AAPC = -2,84\%; IC95\% -3,25 - -2,43).

CONCLUSÕES: Embora as reduções na mortalidade ainda sejam discretas, indicam progresso nas ações de controle do câncer de próstata. Ações de rastreamento e mudanças nas condutas terapêuticas nas últimas décadas podem estar modificando a incidência e a sobrevida, resultando em mudanças no perfil de mortalidade. Estudos mais detalhados serão úteis na compreensão dos fatores que levam às variações inter-regionais encontradas.

DESCRITORES: Neoplasias da Próstata, mortalidade. Mortalidade, tendências. Distribuição por Idade. Distribuição Temporal. 


\section{INTRODUÇÃO}

Em todo o mundo, o câncer de próstata é o segundo mais incidente e a sexta principal causa de morte por câncer em homens ${ }^{1}$, mas as tendências da incidência e mortalidade pela doença variam nos diversos países. As variações geográficas das taxas de incidência são provavelmente decorrentes da combinação de casos prevalentes subjacentes e de diferenças nas práticas relacionadas ao rastreamento, incluindo o exame de PSA (antígeno prostático específico) ${ }^{2,3}$.

O Brasil apresenta grande extensão territorial e disparidades regionais, resultando em diferentes padrões de ocorrência de doenças, entre elas o câncer. Estima-se que, no país, o câncer de próstata seja o tipo de câncer mais incidente em homens, com número esperado de 68.220 casos novos da doença em 2018 e 2019. Este número corresponde a 31,7\% de todos os cânceres (exceto o de pele não melanoma) e às taxas de incidência bruta e ajustada por idade de 66,1 e 66,8 casos novos/100 mil homens, respectivamente. Para o estado de São Paulo, nesse mesmo ano, estimaram-se 14.890 casos novos e uma taxa de incidência ajustada de 59,8 casos novos/100 mil homens ${ }^{4}$. A taxa de mortalidade por câncer de próstata (ajustada por idade), no Brasil, no período de 2006 a 2010, foi de 13,7/100 mil homens, com projeção de 12,9/100 mil para o período de 2011 a 2015. Para a região Sudeste, essas taxas foram de 12,9 e 11,3/100 mil nos respectivos períodos ${ }^{5}$.

No que se refere ao atendimento oncológico pelo Sistema Único de Saúde (SUS), há evidências de que o acesso ao diagnóstico e tratamento se distribui de forma heterogênea em São Paulo 6 , sugerindo a existência de padrões distintos de mortalidade por câncer. Este estudo teve como objetivo estimar a magnitude e identificar padrões de mudança na mortalidade por câncer de próstata em geral e segundo faixas etárias a partir dos 50 anos de idade, no estado de São Paulo e em cada uma de suas 17 redes regionais de atenção à saúde (RRAS), no período entre 2000 e 2015.

\section{MÉTODOS}

Trata-se de um estudo ecológico, que analisou a série de dados no período de 2000 a 2015. Os óbitos por câncer de próstata constituíram o objeto de estudo. Selecionaram-se aqueles ocorridos entre residentes nas 17 RRAS do estado de São Paulo e no estado como um todo. A divisão do território em 17 RRAS, feita em 2012, teve como objetivo principal garantir a universalidade e integralidade da assistência à saúde a toda população paulista. As RRAS são definidas como arranjos organizativos de ações e serviços de saúde para integrar serviços e organizar sistemas e fluxos de informações, oferecendo suporte às atividades de planejamento e definição de fluxos no território?.

Os dados sobre óbitos foram coletados na página eletrônica do Departamento de Informática do Sistema Único de Saúde (Datasus), por meio de download dos arquivos provenientes do Sistema de Informações sobre Mortalidade do Ministério da Saúde ${ }^{8}$. As variáveis selecionadas foram: faixa etária, local de residência, ano e causa básica de morte classificada com o código C61 da 10ª revisão da Classificação Internacional de Doenças 9 . A população masculina residente no estado de São Paulo foi obtida por meio da tabulação de informações sobre estimativas populacionais ${ }^{10}$ para o período entre 2000 e 2015 na página eletrônica do Datasus. Para fazer a representação espacial das taxas de mortalidade no último período da série, foi utilizado o programa Tab para Windows (Tabwin versão 3.6 b) ${ }^{11}$.

Os óbitos foram agregados em grupos de idade quinquenais (de 0 a 80 anos ou mais) e decenais (de 50 a 80 anos ou mais). Calculamos as taxas de mortalidade ajustadas por idade tendo como base 100 mil homens. $\mathrm{O}$ ajuste por idade foi feito pelo método direto, utilizando como padrão a população mundial de Segi ${ }^{12}$. Para representar a magnitude da mortalidade, as taxas foram calculadas para três períodos compreendidos entre $2000 \mathrm{e}$ 2015: período 1 (2000-2005), período 2 (2006-2010) e período 3 (2011-2015). Para a análise de tendência temporal, calculamos as taxas para cada ano da série e para quatro grupos etários (50-59, 60-69, 70-79 e 80 anos ou mais) 
A avaliação da tendência temporal foi realizada pela análise de regressão Joinpoint para identificar os pontos de mudança em um período e para verificar a significância estatística das tendências das taxas ajustadas de mortalidade ${ }^{13}$. Estimamos as medidas síntese da análise de tendência, denominadas de variação percentual anual média (AAPC) ${ }^{14}$ que apontassem a direção (aumento ou redução) e a magnitude das mudanças no período de 2000 a 2015 e em faixas etárias a partir dos 50 anos, acompanhadas dos respectivos intervalos de confiança de 95\% (IC95\%), com o emprego do software Joinpoint Regression Program, versão 4.2.0.1 ${ }^{15}$. Mantivemos a opção padrão do programa para ajuste de um modelo de erros não correlacionados, após testes da presença de autocorrelação serial indicarem que a análise apresentada é segura contra erros de interpretação.

Por se tratar de um estudo ecológico, a coleta de dados foi realizada em bancos de dados de domínio público, não havendo necessidade de submissão do projeto a um comitê de ética em pesquisa.

\section{RESULTADOS}

No período de 2000 a 2015, ocorreram 40.631 óbitos por câncer de próstata entre homens residentes no estado de São Paulo (Tabela 1). No primeiro período (2000-2005), a taxa ajustada

Tabela 1. Redes regionais de atenção à saúde (RRAS) segundo departamento regionais de saúde (DRS) e regiões de saúde correspondentes, população masculina residente em 2015, número de óbitos e taxas de mortalidade por câncer de próstata ajustadas por idade entre 2000 e 2015.

\begin{tabular}{|c|c|c|c|c|c|c|}
\hline \multirow{2}{*}{ RRAS } & \multirow{2}{*}{$\begin{array}{c}\text { DRS } \\
\text { (região de saúde) }\end{array}$} & \multirow{2}{*}{$\begin{array}{l}\text { População masculina } \\
\text { residente (2015) }\end{array}$} & \multirow{2}{*}{$\begin{array}{l}\text { Número de óbitos } \\
(2000-2015)\end{array}$} & \multicolumn{3}{|c|}{$\begin{array}{l}\text { Taxas de mortalidade ajustadas por idade } \\
\text { (por } 100 \text { mil homens) }\end{array}$} \\
\hline & & & & 2000-2005 & 2006-2010 & 2011-2015 \\
\hline 1 & $\begin{array}{l}\text { Grande São Paulo } \\
\text { (Grande ABC) }\end{array}$ & 1.327 .156 & 2.263 & 15,7 & 13,1 & 12,7 \\
\hline 2 & $\begin{array}{l}\text { Grande São Paulo } \\
\text { (Guarulhos e Alto do Tietê) }\end{array}$ & 1.431 .263 & 1.908 & 16,2 & 14,8 & 14,0 \\
\hline 3 & $\begin{array}{l}\text { Grande São Paulo } \\
\text { (Franco da Rocha) }\end{array}$ & 290.202 & 354 & 17,4 & 14,8 & 15,1 \\
\hline 4 & Grande São Paulo (Mananciais) & 539.967 & 635 & 18,3 & 14,4 & 13,3 \\
\hline 5 & $\begin{array}{l}\text { Grande São Paulo } \\
\text { (Rota dos Bandeirantes) }\end{array}$ & 898.382 & 1.241 & 17,7 & 15,4 & 14,6 \\
\hline 6 & $\begin{array}{c}\text { Grande São Paulo } \\
\text { (Município de São Paulo) }\end{array}$ & 5.739 .347 & 11.658 & 16,8 & 14,7 & 12,2 \\
\hline 7 & Baixada Santista e Registro & 1.012 .305 & 2.347 & 15,1 & 15,5 & 13,9 \\
\hline 8 & Sorocaba & 1.225 .859 & 2.144 & 14,2 & 13,2 & 11,5 \\
\hline 9 & Bauru & 878.978 & 1.852 & 14,2 & 12,3 & 11,4 \\
\hline 10 & Marília & 566.350 & 1.245 & 13,6 & 11,1 & 9,3 \\
\hline 11 & Presidente Prudente & 385.065 & 861 & 12,4 & 12,0 & 10,3 \\
\hline 12 & $\begin{array}{c}\text { Araçatuba } \\
\text { São José do Rio Preto }\end{array}$ & 1.180 .992 & 2.346 & 12,1 & 10,0 & 9,3 \\
\hline 13 & $\begin{array}{c}\text { Araraquara, Barretos, Franca e } \\
\text { Ribeirão Preto }\end{array}$ & 1.780 .446 & 3.591 & 14,4 & 13,5 & 11,9 \\
\hline 14 & Piracicaba & 764.711 & 1.386 & 13,5 & 11,6 & 11,5 \\
\hline 15 & $\begin{array}{c}\text { Campinas e São João da Boa Vista } \\
\text { (Rio Pardo, Mantiqueira, Baixa Mogiana, } \\
\text { Oeste VII, Campinas) }\end{array}$ & 2.005 .472 & 3.543 & 15,1 & 11,8 & 11,1 \\
\hline 16 & $\begin{array}{c}\text { Campinas } \\
\text { (Bragança e Jundiaí) }\end{array}$ & 612.182 & 1.136 & 15,4 & 12,3 & 11,4 \\
\hline 17 & Taubaté & 1.222 .358 & 2.121 & 14,1 & 13,7 & 13,2 \\
\hline & Estado de São Paulo & 21.861.035 & 40.631 & 15,2 & 13,3 & 11,9 \\
\hline
\end{tabular}

Fontes: população: estimativas populacionais Rede Interagencial de Informações para a Saúde (RIPSA/Ministério da Saúde ${ }^{10}$; óbitos: Sistema de Informações sobre Mortalidade do Ministério da Saúde (Datasus). 


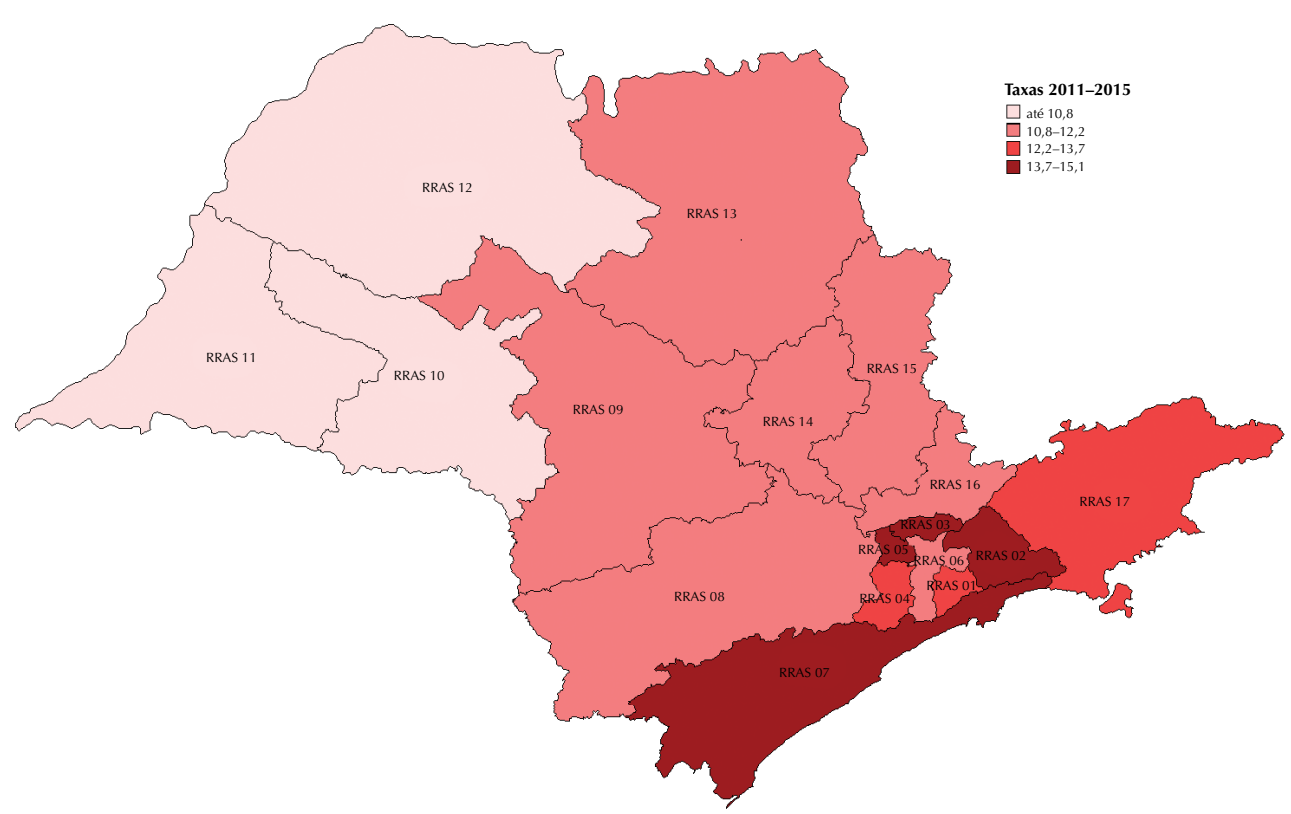

Figura 1. Taxas de mortalidade ajustadas por idade (100 mil homens) por câncer de próstata segundo as 17 redes regionais de atenção à saúde do estado de São Paulo no período de 2011 a 2015.

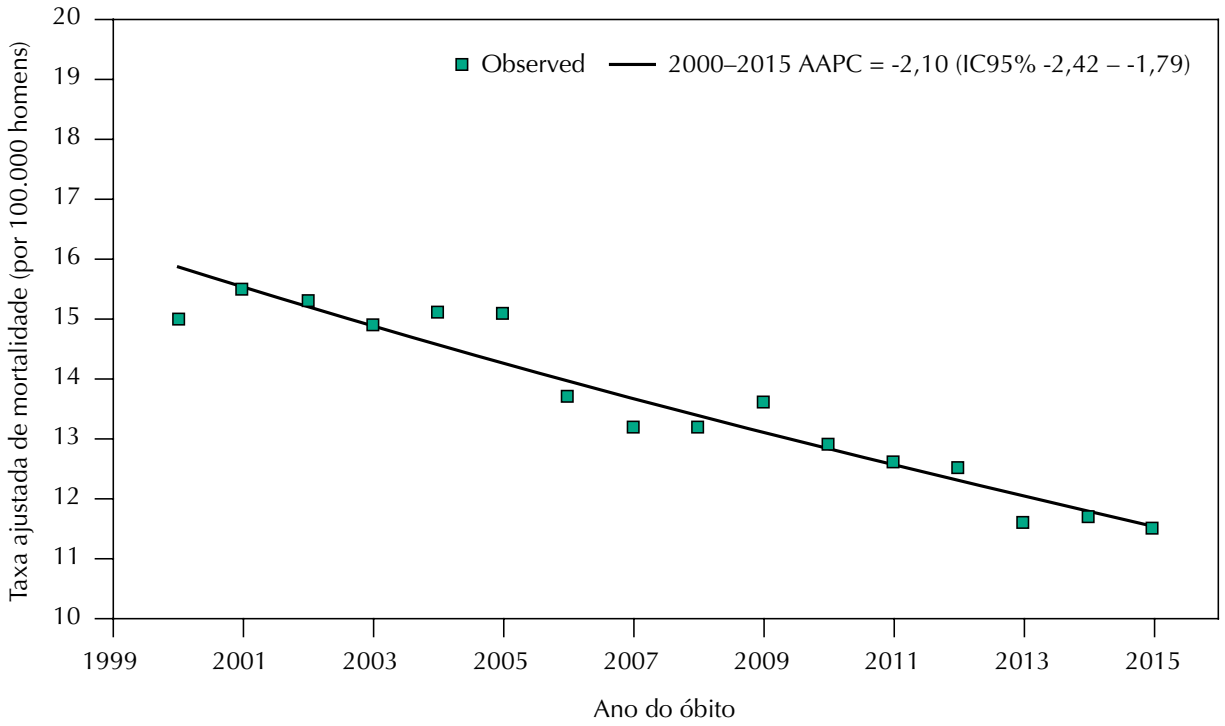

Figura 2. Tendência das taxas de mortalidade (ajustadas por idade) por câncer de próstata no estado de São Paulo no período de 2000 a 2015.

de mortalidade foi de 15,2 óbitos/100 mil homens. Entre as RRAS, as taxas variaram entre 12,1/100 mil (RRAS 12) e 18,3/100 mil (RRAS 4). Nos períodos subsequentes, as taxas para o estado foram de 13,3 e 11,9/100 mil em 2006-2010 e 2011-2015, respectivamente. No último período as taxas mais elevadas foram observadas nas RRAS 3 e 5 (15,1 e 14,6/100 mil) e as mais baixas, nas RRAS 10 e 12 (9,3/100 mil) (Figura 1).

No período estudado, observou-se um decréscimo estatisticamente significativo nas taxas de mortalidade por câncer de próstata no estado de São Paulo (AAPC = -2,10\%; IC95\% -2,42 - -1,79) (Figura 2). Em relação à distribuição regional, observou-se a mesma tendência em 11 das 17 regiões, com reduções médias anuais entre -1,72\% (IC95\% -2,46--0,98), na RRAS 13, e -3,05\% (IC95\% -4,95 - -1,10), na RRAS 10. Nas demais regiões, observou-se tendência de redução sem significância estatística (Tabela 2). 
Tabela 2. Tendência das taxas de mortalidade (ajustadas por idade) por câncer de próstata nas 17 redes regionais de atenção à saúde (RRAS) no período de 2000 a 2015.

\begin{tabular}{lll}
\hline RRAS & AAPC & IC95\% \\
\hline 1 & $\mathbf{- 2 , 0 8}$ & $-2,93--1,22$ \\
2 & $-1,05$ & $-2,15-0,06$ \\
3 & $-1,27$ & $-3,49-1,00$ \\
\hline 4 & $\mathbf{- 2 , 7 7}$ & $-4,16--1,36$ \\
\hline 5 & $\mathbf{- 2 , 4 1}$ & $-4,21--0,59$ \\
\hline 6 & $\mathbf{- 2 , 9 6}$ & $-3,31--2,60$ \\
\hline 7 & $-0,60$ & $-1,60-0,42$ \\
\hline 8 & $\mathbf{- 1 , 8 9}$ & $-3,48--0,28$ \\
\hline 9 & $\mathbf{- 1 , 9 0}$ & $-3,20-0,58$ \\
\hline 10 & $\mathbf{- 3 , 0 5}$ & $-4,95--1,10$ \\
11 & $-1,26$ & $-3,02-0,52$ \\
12 & $-\mathbf{2 , 4 4}$ & $-3,21--1,66$ \\
13 & $\mathbf{- 1 , 7 2}$ & $-2,46--0,98$ \\
14 & $-1,28$ & $-2,91-0,37$ \\
15 & $\mathbf{- 2 , 7 5}$ & $-3,77--1,72$ \\
16 & $\mathbf{- 2 , 6 3}$ & $-4,15--1,07$ \\
17 & $-0,52$ & $-1,51-0,49$ \\
\hline AAPC: & -92 & \\
\hline
\end{tabular}

AAPC: variação percentual anual média; IC95\%: intervalo de 95\% de confiança

Nota: AAPC estatisticamente diferentes de zero em negrito

Tabela 3. Tendência das taxas de mortalidade (ajustadas por idade) por câncer de próstata segundo grupos etários nas 17 redes regionais de atenção à saúde (RRAS) e no estado de São Paulo (ESP) no período de 2000 a 2015.

\begin{tabular}{|c|c|c|c|c|}
\hline \multirow{2}{*}{ RRAS } & \multicolumn{4}{|c|}{ AAPC (IC95\%) } \\
\hline & 50 a 59 anos & 60 a 69 anos & 70 a 79 anos & 80 anos ou mais \\
\hline 1 & $-1,10(-4,56-2,48)$ & $-2,23(-4,99-0,62)$ & $-1,53(-2,94--0,09)$ & $-2,71(-4,36--1,04)$ \\
\hline 2 & $0,41(-3,29-4,25)$ & $-4,03(-6,85--1,12)$ & $0,29(-1,46-2,07)$ & $-0,91(-2,92-1,15)$ \\
\hline 3 & $-*$ & $-5,90(-9,62--2,03)$ & $3,26(0,19-6,41)$ & $-2,84(-7,18-1,70)$ \\
\hline 4 & $-*$ & $-3,98(-9,30-1,65)$ & $-2,79(-7,33-1,97)$ & $-2,98(-5,22--0,68)$ \\
\hline 5 & $-6,04(-11,02--0,79)$ & $-2,67(-5,60-0,34)$ & $-1,79(-4,08-0,54)$ & $-2,33(-4,62-0,02)$ \\
\hline 6 & $-1,92(-4,05-0,25)$ & $-2,77(-3,38--2,16)$ & $-3,47(-4,30--2,63)$ & $-3,43(-4,86--1,97)$ \\
\hline 7 & $-1,58(-7,03-4,18)$ & $-1,75(-3,33--0,15)$ & $-0,68(-2,21-0,88)$ & $0,30(-1,62-2,26)$ \\
\hline 8 & $1,16(-15,42-20,98)$ & $1,12(-8,11-11,27)$ & $-0,75(-2,17-0,70)$ & $-1,87(-4,03-0,35)$ \\
\hline 9 & $-0,59(-5,70 ; 4,80)$ & $-2,77(-5,05--0,44)$ & $-1,88(-3,59--0,15)$ & $-1,82(-3,65-0,05)$ \\
\hline 10 & $-a^{a}$ & $-4,44(-7,09--1,72)$ & $-2,47(-5,16-0,30)$ & $-2,55(-4,75--0,29)$ \\
\hline 11 & $-a^{a}$ & $-3,07(-6,04--0,01)$ & $-1,50(-3,76-0,80)$ & $0,32(-2,04-2,73)$ \\
\hline 12 & $-2,52(-7,23-2,43)$ & $-2,40(-3,29--1,50)$ & $-3,24(-4,31--2,16)$ & $-1,42(-3,01-0,19)$ \\
\hline 13 & $-0,51(-3,20-2,25)$ & $-3,65(-5,23--2,05)$ & $-0,99(-1,96--0,01)$ & $-1,58(-2,94--0,20)$ \\
\hline 14 & $-*$ & $-1,30(-4,31-1,81)$ & $-1,18(-3,35-1,03)$ & $-1,83(-4,38-0,79)$ \\
\hline 15 & $-4,79(-6,99--2,55)$ & $-3,44(-5,23--1,61)$ & $-2,02(-3,40--0,62)$ & $-2,74(-4,14--1,32)$ \\
\hline 16 & $-4,40(-8,61-0,00)$ & $-2,18(-5,00-0,73)$ & $-2,12(-4,64-0,47)$ & $-2,95(-4,79--1,08)$ \\
\hline 17 & $-4,41(-9,08-0,49)$ & $-3,93(-6,15--1,65)$ & $0,26(-1,30-1,84)$ & $0,57(-1,55-2,73)$ \\
\hline ESP & $-2,33(-3,04--1,62)$ & $-2,84(-3,25--2,43)$ & $-1,93(-2,36--1,50)$ & $-1,92(-2,40--1,44)$ \\
\hline
\end{tabular}

AAPC: variação percentual anual média; IC95\%: intervalo de $95 \%$ de confiança

Nota: AAPC estatisticamente diferentes de zero em negrito

* A AAPC não foi calculada pois não houve registro de óbito por câncer de próstata em pelo menos um ano da série entre 2000 e 2015. 
Entre 2000 e 2015, 99\% dos óbitos por câncer de próstata ocorreram a partir dos 50 anos. As taxas de mortalidade específicas por idade mostraram grande variação e aumento do risco de morrer com o avançar da idade. Para o estado de São Paulo, no último ano da série, as taxas de mortalidade para os grupos quatro decenais compreendidos entre 50 e 80 anos ou mais foram, respectivamente, de 5,5, 34,4, 152,5 e 408,8 óbitos/100 mil homens.

A análise de tendência segundo faixas etárias indicou redução estatisticamente significativa da mortalidade em todos os grupos a partir dos 50 anos para o estado (Tabela 3), com os maiores percentuais de redução nas faixas de 50 a 59 (AAPC =-2,33; IC95\% -3,04 - -1,62) e de 60 a 69 anos (AAPC $=-2,84$; IC95\% $-3,25--2,43)$.

$\mathrm{Na}$ análise estratificada por regiões, no grupo de 50 a 59 anos, observaram-se reduções significativas na RRAS 5 (AAPC = -6,04; IC95\% -11,02 - -0,79) e na RRAS 15 (AAPC = -4,79; IC95\% -6,99 - -2,55). No grupo de 60 a 69 anos, em 11 das 17 RRAS, verificaram-se reduções significativas na mortalidade, principalmente na RRAS 2 e na RRAS 3. No grupo de 70 a 79 anos, o maior declínio (AAPC $=-3,47$; IC95\% -4,30 - -2,63) foi observado na RRAS 6 e reduções significativas, de pelo menos 0,99\%, em outras cinco RRAS. Na faixa de 80 anos ou mais, decréscimos significativos foram observados em sete regiões, sendo que a RRAS 6 apresentou a redução de maior magnitude (AAPC = -3,43; IC95\% -4,86 - -1,97) (Tabela 3).

\section{DISCUSSÃO}

No período de 2000 a 2015, houve um decréscimo estatisticamente significativo das taxas de mortalidade por câncer de próstata no estado de São Paulo e em 11 das 17 RRAS. Mundialmente a tendência temporal de redução da mortalidade por esse tipo de câncer foi encontrada em áreas desenvolvidas como Reino Unido (-1,14\% a cada ano entre 1992 e 2004) e Estados Unidos (-4,17\% entre 1994 e $2004^{16}$ e -7,19\% entre 2009 e 2013) $)^{17}$. Para o Brasil e suas regiões, entre 1980 e 2010, observou-se tendência ascendente em um estudo que incluiu a redistribuição de óbitos por causas mal definidas na correção das taxas de mortalidade ${ }^{18}$.

No estado de São Paulo, no período de 2011 a 2015 a taxa de mortalidade por câncer de próstata ajustada por idade (11,9 óbitos/100 mil homens) foi inferior ao risco de morte estimado para a América do Sul em 2018 (14/100 mil), superior ao estimado para América do Norte, Sul da Europa e Ásia Ocidental (8/100 mil), e similar ao estimado para Reino Unido (13/100 mil), Colômbia e Argentina (12/100 mil) ${ }^{1}$. Globalmente as taxas de mortalidade por câncer de próstata apresentam menor variabilidade geográfica do que a incidência da doença ${ }^{1}$. Os maiores riscos de morte são observados em regiões menos desenvolvidas e onde há predomínio de população negra, como nas regiões do Caribe e África Subsaariana (taxas de 23 a 27/100 mil)1. Quanto à tendência temporal, declínios foram notados previamente em áreas de recursos mais elevados e aumento em países de poucos recursos².

Estudos de mortalidade são essenciais para indicar prioridades em saúde pública; entretanto, as interpretações a partir dessa medida isolada podem ser falaciosas. Tendências da mortalidade por câncer são resultado de tendências anteriores tanto da incidência como da sobrevida ${ }^{19}$. No estado de São Paulo, assim como no Brasil, a incidência de câncer está disponível para poucos municípios cobertos por registros de câncer de base populacional (RCBP), ou é produzida por meio de estimativas para estados e capitais pelo Instituto Nacional do Câncer desde $1995^{4}$. Por questões metodológicas, essas estimativas não devem ser utilizadas para estudos de séries temporais. Apesar de existirem cinco RCBP em atividade no estado, que fornecem dados de incidência para seus municípios de cobertura ${ }^{20}$, não foi possível neste estudo avaliar os efeitos das taxas de incidência e sobrevida do câncer de próstata na mortalidade pela doença, seja pela pequena representatividade populacional no âmbito estadual, seja pela instabilidade nas taxas ao longo do tempo. 
Não há evidências científicas consistentes demonstrando que o rastreamento com teste do PSA reduza a mortalidade ${ }^{21}$ pelo câncer de próstata. Ações de rastreamento produzem efeitos de curto prazo nas taxas de incidência pela detecção de tumores que não teriam sido diagnosticados clinicamente ou que não causariam mortes ${ }^{19}$. Nos Estados Unidos, onde o rastreamento para o câncer de próstata foi introduzido nos anos 1990, observou-se declínio da mortalidade no início da mesma década ${ }^{16}$; no entanto, alguns autores afirmam que seria precoce atribuir a redução da mortalidade aos efeitos do rastreamento ${ }^{16,22}$, pois a maior parte do declínio observado pode ser devida a outros fatores, especialmente à melhora no tratamento ${ }^{16}$.

Dados do Sistema de Informações Ambulatoriais do SUS mostraram aumento no número de exames de PSA ${ }^{23}$ realizados por estabelecimentos de saúde localizados no estado de São Paulo. Entre 2008 e 2018, verificou-se incremento de $94 \%$ e número médio de exames anuais de $1.368 .695^{23}$. Entre os residentes no estado, dados parciais do sistema de informação em saúde que dispunha da informação sobre o local de residência ${ }^{24}$ mostraram maiores volumes em 2016 e 2018 (respectivamente de 10.378 e 8.943 exames), ao passo que em 2014, 2015 e 2017, os volumes dos exames foram mais baixos (1.406, 2.506 e 4.372, respectivamente), não indicando aumento consistente na realização de exames.

Acerca da detecção precoce da doença, não existem dados de base populacional sobre o estadiamento no momento do diagnóstico dos tumores de próstata entre residentes no estado de São Paulo, os quais poderiam suscitar hipóteses sobre as diferenças regionais apontadas nos achados deste estudo. Segundo o Registro Hospitalar de Câncer de São Paulo ${ }^{25}$, dados de 64.745 tumores invasivos de próstata estadiáveis pela classificação $\mathrm{TNM}^{26-28}$ diagnosticados entre residentes no estado entre 2000 e 2014 mostraram redução na proporção de casos com extensão extraprostática ao diagnóstico, isto é, casos em estádios avançados (III e IV). Nos períodos de 2000-2004, 2005-2009 e 2010-2014, as proporções de estádios avançados foram, respectivamente, de 41,7\%, 30,6\% e 28,7\% ${ }^{25}$. A redução na proporção de estádios III e IV também foi observada em residentes de 16 das 17 RRAS, exceto pela RRAS 3, onde as proporções foram 31,3\%, 24,6\% e 34,5\% nos referidos períodos. Embora tenha havido redução na proporção de casos diagnosticados em estádios avançados em quase todas as RRAS, esses achados sugerem a existência de outros fatores que estariam relacionados aos diferenciais de mortalidade apontados neste estudo, entre eles o acesso ao diagnóstico e tratamento oncológico e mudanças nas condutas terapêuticas nas últimas décadas.

Em relação aos efeitos do tratamento oncológico sobre as taxas de mortalidade, é de se destacar que a mortalidade constitui-se como indicador pouco preciso na comparação entre grupos de pacientes com prognósticos muito diferentes, pois as mortes ocorridas em um determinado ano não se referem às mortes entre pacientes que foram diagnosticados em torno do mesmo período e, possivelmente, teriam recebido tratamento oncológico semelhante. Assim, essa medida reflete tardiamente o efeito de progressos no controle do câncer quando se trata de avaliar como as mudanças no diagnóstico e tratamento influenciam o prognóstico ${ }^{19}$.

Identificamos ainda reduções anuais significativas na mortalidade em todos os grupos etários a partir dos 50 anos no estado de São Paulo. Nas demais regiões decréscimos também foram observados, principalmente no grupo de 60 a 69 anos. Considerando que há elevada proporção de causas mal definidas nas idades avançadas ${ }^{29}$, a análise temporal da mortalidade por grupos de idade poderia estar comprometida se as reduções observadas fossem resultantes de piora na qualidade do preenchimento das declarações de óbito. Apesar de 74\% dos óbitos masculinos por causas mal definidas entre residentes no estado estarem concentrados em grupos etários a partir dos $50 \operatorname{anos}^{30}$, no período de 2000 a 2015, foram verificados baixos percentuais anuais (7,5\% em 2000 e 4,9\% em 2015). Entre as regiões, em 2000, o percentual de causas mal definidas variou de $1 \%$ (RRAS 5 e 6) a 18\% (RRAS 7 e 10); em 2015 , os percentuais foram de $1 \%$ a $13 \%$, com percentual inferior a $10 \%$ em 13 regiões ${ }^{30}$. 
Nas cinco RRAS menos populosas, no grupo de homens entre 50 e 59 anos, não foram registrados óbitos por câncer de próstata em algum ano entre 2000 e 2015. Embora apenas $5 \%$ dos óbitos por câncer de próstata tenham ocorrido em indivíduos nessa faixa etária ${ }^{8}$ no período de 2011 a 2015, consideramos relevante apresentar a tendência temporal das taxas de mortalidade por grupos de idade, permitindo comparar a mortalidade prematura pela doença com a mortalidade em idades mais avançadas para possível indicação de medidas prioritárias em regiões e/ou grupos etários específicos. Ponderamos também que o poder estatístico da análise estaria reduzido pela distribuição dos óbitos totais para o estado por RRAS de residência, ocasionando flutuação no número absoluto de mortes ano a ano. Entretanto, consideramos válido apresentar a tendência da mortalidade por regiões e grupos etários, pois os subgrupos mais populosos e com maior estabilidade na ocorrência das mortes mostrariam significância estatística e indicariam aspectos locais passíveis de ações.

Como limitações do estudo, podemos destacar que a ausência de dados de base populacional para análise conjunta com as estatísticas de mortalidade não permitiu que a mortalidade diferencial entre regiões e grupos etários fosse melhor explorada. Outra limitação refere-se à qualidade do preenchimento da causa básica de morte. Embora o estado de São Paulo apresente adequada validade dos dados, indicada pelas baixas proporções de óbitos por causas mal definidas e tendência de redução progressiva ao longo dos $\operatorname{anos}^{30}$, pode-se considerar que a qualidade de preenchimento das declarações de óbito não é uniforme ao longo da série temporal e também entre as RRAS.

Os achados obtidos neste estudo evidenciam a redução na mortalidade pelo tipo de câncer mais incidente em homens. Embora as reduções observadas ainda sejam discretas, indicam progresso nas ações de controle do câncer de próstata na população do estado de São Paulo. Ações de rastreamento e mudanças nas condutas terapêuticas nas últimas décadas constituem fatores que estariam modificando padrões de incidência e de sobrevida pelo câncer de próstata na população paulista, resultando nas mudanças nas taxas de mortalidade no período de 2000 a 2015. Em um contexto de limitada disponibilidade de dados sobre incidência, ressalta-se a importância da utilização das estatísticas de mortalidade como complemento do perfil de morbidade por câncer.

Estudos epidemiológicos mais detalhados serão úteis na identificação e compreensão dos fatores que levam às variações inter-regionais encontradas, incluindo dados de acesso aos serviços de saúde. Algumas hipóteses aventadas explicariam parte dessas diferenças. Cabe ainda ressaltar que investimentos em registros de câncer de base populacional devem surtir efeitos positivos na produção de dados completos e de qualidade para subsidiar políticas e ações específicas, bem como contribuir para a análise da ocorrência do câncer ao longo do tempo.

\section{REFERÊNCIAS}

1. Ferlay J, Ervik M, Lam F, Colombet M, Mery L, Piñeros M, et al, editors. Cancer Today (powered by GLOBOCAN 2018). Lyon (FR); France: International Agency for Research on Cancer; 2018 [citado 21 maio 2019]. (IARC CancerBase, $\mathrm{n}^{\circ}$ 15). Disponível em: https://gco.iarc.fr/today

2. Center MM, Jemal AJ, Lortet-Tieulent J, Ward E, Ferlay J, Brawley O, et al. International variation in prostate cancer incidence and mortality rates. Eur Urol. 2012;61(6):1079-92. https://doi.org/10.1016/j.eururo.2012.02.054

3. Schröder FH, Roobol MJ. Prostate cancer epidemic in sight? Eur Urol 2012;61(6):1093-5. https://doi.org/10.1016/j.eururo.2012.03.019

4. Instituto Nacional de Câncer José Alencar Gomes da Silva, Coordenação de Prevenção e Vigilância. Estimativa 2018: incidência de câncer no Brasil. Rio de Janeiro: INCA; 2017.

5. Jerez-Roig J, Souza DLB, Medeiros PFM, Barbosa IR, Curado MP, Costa ICC, et al. Future burden of prostate cancer mortality in Brazil: a population-based study. Cad Saude Publica. 2014;30(11):2451-8. https://doi.org/10.1590/0102-311X00007314 
6. Fundação Oncocentro de São Paulo; Secretaria de Estado da Saúde de São Paulo. Caracterização da assistência oncológica nas Redes Regionais de Atenção à Saúde no estado de São Paulo em 2010: relatórios técnicos. São Paulo; 2014 [citado 10 jul 2017]. (RRAS, 1 a 17). Disponível em: http://www.fosp.saude.sp.gov.br/publicacao/boletinsraas

7. Ministério da Saúde (BR). Portaria n. ${ }^{\circ} 4.279$, de 30 de dezembro de 2010. Estabelece diretrizes para a organização da Rede de Atenção à Saúde no âmbito do Sistema Único de Saúde (SUS). Diário Oficial da União. 31 dez 2010; Seção 1:89.

8. Ministério da Saúde (BR), Departamento de Informática do Sistema Único de Saúde - DATASUS. Banco de Dados do Sistema de Informação sobre Mortalidade - SIM. Brasília, DF: DATASUS; 2018 [citado 10 dez 2018]. Disponível em: http://www.datasus.gov.br

9. Organização Mundial da Saúde. Classificação Estatística Internacional de Doenças e Problemas Relacionados à Saúde -CID-10. 10. rev. São Paulo: EDUSP; 1997.

10. Ministério da Saúde (BR), Departamento de Informática do Sistema Único de Saúde DATASUS. População residente: estimativas populacionais elaboradas pela RIPSA e Ministério da Saúde, 2000 a 2015. Brasília, DF: DATASUS; 2015 [17 maio 2019]. Disponível em: http://tabnet.datasus.gov.br/cgi/deftohtm.exe?novapop/cnv/popbr.def

11. Ministério da Saúde (BR), Departamento de Informática do Sistema Único de Saúde. TabWin. Tab para Win 32 ou Wine. Versão 3.6. Brasília, DF: DATASUS; 2019 [citado 21 maio 2019]. Disponível em: http://datasus1.saude.gov.br/transferencia-download-de-arquivos/download-do-tabwin

12. Doll R, Cook P. Summarizing indices for comparison of cancer incidence data. Int J Cancer.1967;2(3):269-79. https://doi.org/10.1002/ijc.2910020310

13. Kim HJ, Fay MP, Feuer EJ, Midthune DN. Permutation tests for joinpoint regression with applications to cancer rates. Stat Med. 2000;19:335-51. https://doi.org/10.1002/(sici)1097-0258(20000215)19:3<335::aid-sim336>3.0.co;2-z

14. Clegg LX, Hankey BF, Tiwari R, Feuer EJ, Edwards, BK. Estimating average annual percent change in trend analysis. Stat Med 2009;28(29):3670-82. https://doi.org/10.1002/sim.3733

15. National Cancer Institute, Division of Cancer Control and Popuation Sciences. Joinpoint Regression Program, version 4.2.0.1 (May 2015). Bethesda, MA: Survellance Reseach Program, Statistical Methodology and Applications Branch; 2015.

16. Collin SM, Martin RM, Metcalfe C, Gunnell D, Albertsen PC, Neal D, et al. Prostate-cancer mortality in the USA and UK in 1975-2004: an ecological study. Lancet Oncol. 2008;9(5):44552. https://doi.org/10.1016/S1470-2045(08)70104-9

17. Jemal A, Ward EM, Johnson CJ, Cronin KA, Ma J, RyersonAB, et al. Annual report to the nation on the status of cancer, 1975-2014, featuring survival. J Natl Cancer Inst. 2017;109(9):djx030. https://doi.org/10.1093/jnci/djx030

18. Conceição MBM, Boing AF, Peres KG. Time trends in prostate cancer mortality according to major geographic regions of Brazil: an analysis of three decades. Cad Saude Publica. 2014;30(3):559-66. https://doi.org/10.1590/0102-311X00005813

19. Ellis L, Woods LM, Estève J, Eloranta S, Coleman MP, Rachet B. Cancer incidence, survival and mortality: explaining the concepts. Int J Cancer. 2014;135(8):1774-82. https://doi.org/10.1002/ijc.28990

20. Instituto Nacional de Câncer José Alencar Gomes da Silva; Ministério da Saúde (BR), Secretaria de Atenção à Saúde. Tabulador das informações dos RCBP. Rio de Janeiro: INCA; 2019 [citado 24 maio 2019]. Disponível em: https://www.inca.gov.br/BasePoplncidencias/Home.action

21. Ilic D, Neuberger MM, Djulbegovic M, Dahm P. Screening for prostate cancer. Cochrane Database Syst Rev. 2013;(1):CD004720. https://doi.org/10.1002/14651858.CD004720.pub3

22. Etzioni R, Tsodikov A, Mariotto A, Szabo A, Falcon S, Wegelin J et al. Quantifying the role of PSA screening in the US prostate cancer mortality decline. Cancer Causes Control. 2008;19(2):175-81. https://doi.org/10.1007/s10552-007-9083-8

23. Ministério da Saúde (BR), Departamento de Informática do Sistema Único de Saúde DATASUS. Sistema de Informações Ambulatoriais do SUS (SIASUS) - tabulação por local de atendimento - São Paulo. Brasília, DF: DATASUS; 2019 [citado 24 jun 2019]. Disponível em: http://tabnet.datasus.gov.br/cgi/deftohtm.exe?sia/cnv/qasp.def

24. Ministério da Saúde (BR), Departamento de Informática do Sistema Único de Saúde DATASUS. Sistema de Informações Ambulatoriais do SUS (SIASUS) - tabulação por local de residência - São Paulo. Brasília, DF: DATASUS; 2019 [citado 24 jun 2019]. Disponível em: http://tabnet.datasus.gov.br/cgi/deftohtm.exe?sia/cnv/qbsp.def 
25. Fundação Oncocentro de São Paulo; Secretaria de Estado da Saúde de São Paulo. Download de bancos de dados de acesso público. São Paulo; 2019 [citado 10 maio 2019]. Disponível em: http://www.fosp.saude.sp.gov.br/publicacoes/downloadarquivos

26. Instituto Nacional de Câncer José Alencar Gomes da Silva. Coordenação Geral de Prevenção e Vigilância. TNM: classificação de tumores malignos. $5^{a}$ ed. Tradução dos originais em inglês. Rio de Janeiro: Inca; 1998.

27. Instituto Nacional de Câncer José Alencar Gomes da Silva. Coordenação Geral de Prevenção e Vigilância. TNM: classificação de tumores malignos. $6^{a}$ ed. Tradução dos originais em inglês. Rio de Janeiro: Inca; 2004.

28. Instituto Nacional de Câncer José Alencar Gomes da Silva. Coordenação Geral de Prevenção e Vigilância. TNM: classificação de tumores malignos. $7^{a}$ ed. Tradução dos originais em inglês. Rio de Janeiro: Inca; 2012.

29. Mello Jorge MHP, Laurenti R, Lima-Costa MF, Gotlieb SLD, Chiavegatto Filho ADP. A mortalidade de idosos no Brasil: a questão das causas mal definidas. Epidemiol Serv Saude. 2008;17(4):271-81. https://doi.org/10.5123/S1679-49742008000400004

30. Ministério da Saúde (BR), Departamento de Informática do Sistema Único de Saúde DATASUS. Tabulação de estatísticas vitais/ mortalidade geral do SIM/MS - tabulação por local de residência - São Paulo. Brasília, DF; 2019 [citado 24 jun 2019]. Disponível em: http://tabnet. datasus.gov.br/cgi/deftohtm.exe?sim/cnv/obt10sp.def

Financiamento: Fundação de Amparo à Pesquisa do Estado de São Paulo (FAPESP - Projeto Temático - Auxílio à Pesquisa - Processo 2017/03812-7.

Contribuição dos Autores: Concepção e planejamento do estudo: CTML, KBR, JEN. Coleta, análise e interpretação dos dados: CTML, KBR. Elaboração e revisão do manuscrito: CTML, KBR, LAMF, JEN. Aprovação da versão final: CTML, KBR, LAMF, JEN. Responsabilidade pública pelo conteúdo do artigo: CTML, KBR, LAMF, JEN.

Conflito de Interesses: Os autores declaram não haver conflito de interesses. 